INPLASY

PROTOCOL

To cite: Shi et al. Effectiveness of Xuebijing injection for

Sepsis: An Overview of

Systematic Reviews and Meta-

analyses. Inplasy protocol

2020120126. doi:

10.37766/inplasy2020.12.0126

Received: 24 December 2020

Published: 25 December 2020

Corresponding author:

Yu-dong Xu

xydong0303@126.com

Author Affiliation:

Shanghai Research Institute of Acupuncture \& Meridian

Support: NSFC.

Review Stage at time of this submission: Preliminary

searches.

Conflicts of interest:

None.

\section{Effectiveness of Xuebijing injection for Sepsis: An Overview of Systematic Reviews and Meta-analyses}

Shi, YL1; Chen, CT2; Yang, YQ3; Wang, Y4; Chen, YJ5; Xu, YD6.

Review question / Objective: What are the effects of Xuebijing injection (XBJ) for sepsis? Many systematic reviews/metaanalyses have been conducted for XBJ's efficacy, but existed reviews summarized the evidence varied in their quality, intervention dose, control measures and reported outcomes, making the interpretation of the evidence complexed for clinicians and policy makers. In view of this, we plan to use AMSTAR 2 tool and GRADE system to evaluate the methodological quality and evidence quality of existed reviews, so as to provide more reliable evidence of XBJ for sepsis and provide reference for the future improvement of RCTs and SRs/MAs.

INPLASY registration number: This protocol was registered with the International Platform of Registered Systematic Review and Meta-Analysis Protocols (INPLASY) on 25 December 2020 and was last updated on 26 April 2021 (registration number INPLASY2020120126).

\section{INTRODUCTION}

Review question / Objective: What are the effects of Xuebijing injection (XBJ) for sepsis? Many systematic reviews/metaanalyses have been conducted for XBJ's efficacy, but existed reviews summarized the evidence varied in their quality, intervention dose, control measures and reported outcomes, making the interpretation of the evidence complexed for clinicians and policy makers. In view of this, we plan to use AMSTAR 2 tool and GRADE system to evaluate the methodological quality and evidence quality of existed reviews, so as to provide more reliable evidence of $\mathrm{XBJ}$ for sepsis 
and provide reference for the future improvement of RCTs and SRs/MAs.

Condition being studied: Xuebijing injection (XBJ), a Chinese patent medicine, is approved for treating sepsis as a State Category II Drug in China and has been approved by the State Food and Drug Administration of China for clinical practice since 2004. Sepsis is a worldwide public health challenge because of its high prevalence, mortality and disability, leading to significant economic and social burden. Many systematic reviews (SRs) /metaanalyses (MAs) have been conducted to prove the effects of XBJ for sepsis during the past several years. High-quality SRs/ MAs can provide a reliable scientific basis for clinicians, patients, and other decision makers. However, existed SRs/MAs showed varied and heterogeneous results. To summarize the evidence on the effects of XBJ for sepsis, we plan to conduct an overview of existing SRs/MAs.

\section{METHODS}

Search strategy: The four international electronic databases of Pubmed, Embase, Cochrane library, and Web of science and four Chinese electronic databases of China National Knowledge Infrastructure Database (CNKI), WANFANG DATA, Chongqing VIP (CQVIP) and Chinese Biomedical Literature Database (CBM) were searched without language restriction. The basic search strategies were as follows: ("sepsis" OR "severe sepsis" OR "septic shock") AND ("xuebijing" OR "xue bi jing" OR "XBJ") AND ("systematic review" OR "metaanalysis"). Meanwhile, we reviewed the references of included literatures to avoid the occurrence of omission.

Participant or population: Patients who are diagnosed with sepsis by standard diagnostic criteria.

Intervention: Xuebijing plus routine treatment.

Comparator: Routine treatment alone (consist of fluid resuscitation, source control, antibiotic therapy and organ support therapy, et al).

Study designs to be included: Systematic reviews/meta-analyses of randomized controlled trials.

Eligibility criteria: The inclusion criteria were: 1) Patients were diagnosed with sepsis by standard diagnostic criteria; 2) The intervention groups were XBJ plus routine treatment (RT); 3) The control groups were RT alone, and RT comprises fluid resuscitation, source control, antibiotic therapy and organ support therapy; 4) At least one outcome followed was measured: 28-day mortality, Acute Physiology and Chronic Health Evaluation II (APACHE II) scores (the higher the score, the more frequent the need for monitoring and treatment), infection (measured by white blood cells [WBC] or procalcitonin [PCT] or C-Reactive Protein [CRP]), or coagulation function (measured by platelet [PLT] or activated partial thromboplastin time [APTT] or prothrombin time [PT]); 5) SRs/MAs of RCTs.

Information sources: We review the references of included literatures to avoid the occurrence omission. Also, we will contact with author in case that we could not obtain full text.

Main outcome(s): 28-day mortality, Acute Physiology and Chronic Health Evaluation II (APACHE II) scores (the higher the score, the more frequent the need for monitoring and treatment), infection (measured by white blood cells [WBC] or procalcitonin [PCT] or C-Reactive Protein [CRP]), or coagulation function (measured by platelet [PLT] or activated partial thromboplastin time [APTT] or prothrombin time [PT]).

Quality assessment / Risk of bias analysis: The methodological quality of included SRs/MAs will be assessed by the Assessment of Multiple Systematic Reviews 2 (AMSTAR 2). The quality of evidence for outcomes of 28-day mortality, APACHE II scores, WBC, PCT, CRP, PLT, APTT, PT will be assessed using the Grading of Recommendations, 
Assessment, Development and Evaluation (GRADE).

Strategy of data synthesis: We will report the results in text and tables, together with comments on the reliability of the evidence. We will integrate and report data by interventions and major outcomes.

Subgroup analysis: We will conduct subgroup analysis if it is necessary.

Sensibility analysis: Not Applicable.

Country(ies) involved: China.

Keywords: Overview, Xuebijing, Sepsis, Systematic review, Meta-analysis.

Contributions of each author:

Author 1 - Yang-lin Shi.

Email: syl_hnjy@126.com

Author 2 - Cai-tao Chen.

Email: ccttcm@126.com

Author 3 - Yong-qing Yang

Author 4 - Yu Wang.

Author 5 - Yan-jiao Chen.

Author 6 - Yu-dong Xu.

Email: xydong0303@126.com 